\title{
Cirugía Coronaria: Revascularización miocárdica sin circulación extracorpórea*
}

\author{
Dr. ROBERTO GONZÁLEZ L. ${ }^{1}$, ENRIQUE SEGUEL S. ${ }^{1}$, ALECK STOCKINS L. ${ }^{1}$, \\ RODRIGO CAMPOS M. ${ }^{1}$, LUIS NEIRA S. ${ }^{1}$, EMILIO ALARCÓN C. ${ }^{1}$ \\ 1 Equipo de Cirugía Cardiotorácica, Hospital "Dr. Guillermo Grant Benavente”, Facultad de Medicina, \\ Universidad de Concepción, Concepción, Chile.
}

\section{Coronary artery bypass graft without pump oxigenator}

\section{Introducción}

La cirugía de revascularización miocárdica es el patrón de oro en el tratamiento de un amplio espectro de la enfermedad coronaria. Las ventajas de la cirugía con circulación extracorpórea, como operar en un campo inmóvil y sin sangre, llevaron a que la cirugía coronaria con CEC se convirtiera en la técnica de elección. Hoy en día, la cirugía coronaria sin CEC se considera una técnica alternativa de revascularización miocárdica tan segura como la técnica con CEC. El objetivo de esta comunicación es presentar la técnica de la cirugía coronaria sin CEC utilizada por nuestro equipo.

La cirugía de revascularización miocárdica está indicada en el tratamiento de pacientes con enfermedad coronaria, especialmente en aquellos con enfermedad de tronco coronario izquierdo; enfermedad de tres vasos; y enfermedad de uno o dos vasos con compromiso proximal de la arteria descendente anterior ${ }^{1}$. Esta es la cirugía más estudiada de la historia, existe un seguimiento adecuado de sus resultados que se extienden por más de 30 años y es el tratamiento con el cual todas las estrategias de revascularización miocárdica deben ser comparados.

En los inicios de la revascularización miocárdica quirúrgica se realizaron cirugías sin circulación extracorpórea $(\mathrm{CEC})^{2-4}$, pero el rápido desarrollo y las facilidades ofrecidas por la CEC (corazón inmóvil, campo exangüe, adecuada identificación y visualización de los vasos objetivos) llevaron a que ésta se convirtiera en la técnica de elección.

A fines de la década del 80 y principios de los 90, Buffolo y Benetti presentaron sus resultados con grandes series de pacientes en quienes realizaron cirugía coronaria sin $\mathrm{CEC}^{5-7}$. Los buenos resultados y el desarrollo de mejores sistemas de estabilización cardíacos y coronarios, renovaron el interés en la cirugía sin CEC.

La cirugía coronaria sin CEC se considera hoy en día una técnica alternativa de revascularización miocárdica tan segura como la técnica con CEC. Iniciamos su uso en marzo del 2004 y el objetivo de esta comunicación es presentar la técnica que utiliza nuestro equipo.

\section{Técnica quirúrgica}

Todos los pacientes se intervienen con anestesia general por esternotomía media. Luego de la obtención de los conductos necesarios según cada caso, se anticoagula a los pacientes con heparina

*Recibido el 10 de Agosto de 2009 y aceptado para publicación el 2 de Septiembre de 2009.

No existen conflictos de interés ni apoyo financiero

Correspondencia: Dr. Roberto González L.

Janequeo esquina Chacabuco, Concepción, Chile. Fono-Fax: 56-41-2204881

E-mail: rgonzalezlagos@udec.cl 
endovenosa (dosis de 3 miligramos por kilogramo de peso).

Para la estabilización coronaria y del corazón utilizamos estabilizadores por succión OPVAC ${ }^{\circledR}$ y Pyramid ${ }^{\circledR}$ de la empresa Estech ${ }^{\circledR}$ u Octopus ${ }^{\circledR}$ y Starfish ${ }^{\circledR}$ de la empresa Medtronic ${ }^{\circledR}$, según disponibilidad en el hospital.

El perfusionista permanece en todo momento en el pabellón, pero la bomba de circulación extracorpórea sólo se prepara en caso de decidir una conversión a cirugía con CEC. Las razones para la conversión pueden ser anatómicas, como en el caso de vasos intramiocárdicos, muy finos o que no se pueden exponer adecuadamente para la anastomosis, como también la inestabilidad eléctrica o hemodinámica del paciente.

Después de la apertura del pericardio, se instala una compresa húmeda con solución fisiológica tibia detrás del corazón para levantarlo y así exponer mejor la cara anterior. Se explora y selecciona un sitio adecuado para anastomosis en la arteria descendente anterior y se instala el estabilizador coronario para inmovilizar la arteria (Figura 1). Se ocluye la arteria hacia proximal y distal al sitio de anastomosis con torniquetes de polipropileno 5-0. Se realiza la arteriotomía y se procede a la anastomosis témino-lateral de la arteria mamaria interna con sutura continua de polipropileno 7-0. Para ayudar en la exposición y mantener el campo exangüe se utiliza $\mathrm{CO}_{2}$ y solución fisiológica. Una vez terminada la anastomosis se retiran los torniquetes y el clamp de la arteria mamaria, la que comienza a irrigar el corazón inmediatamente (Figura 2). De rutina en nuestro equipo no utilizamos shunt coronarios.

Para exponer la cara lateral se instala el estabilizador cardíaco en la punta del corazón y se levanta cuidadosamente, vigilando la estabilidad hemodinámica del paciente (Figura 3).

Se explora la cara lateral y se identifican las ramas laterales o marginales de la arteria circunfleja. Luego de seleccionar el sitio para anastomosis se estabiliza la arteria con el estabilizador coronario (Figura 4). Se instala un torniquete de polipropileno 5-0 a proximal, se realiza la arteriotomía y la anastomosis término-lateral con vena safena con sutura continua de polipropileno 7-0 (Figura 5). Una vez terminada la sutura se retira el torniquete y se prueba la permeabilidad de la anastomosis inyectando solución fisiológica tibia a través de la vena. De la misma forma se expone la cara inferior con el estabilizador cardíaco, se identifican y estabilizan los objetivos de esa pared con el estabilizador coronario y realizan la anastomosis (Figura 6).

Una vez terminadas las anastomosis distales, se devuelve el corazón a su posición, se mide el largo de los puentes y se instala un clamp lateral en la aorta ascendente para la confección de las anastomosis proximales con sutura continua de polipropileno 6-0.

Se revierte el efecto de la heparina con protamina, se instalan dos tubos de drenaje mediastínico y cables de marcapasos epicárdicos transitorios. El

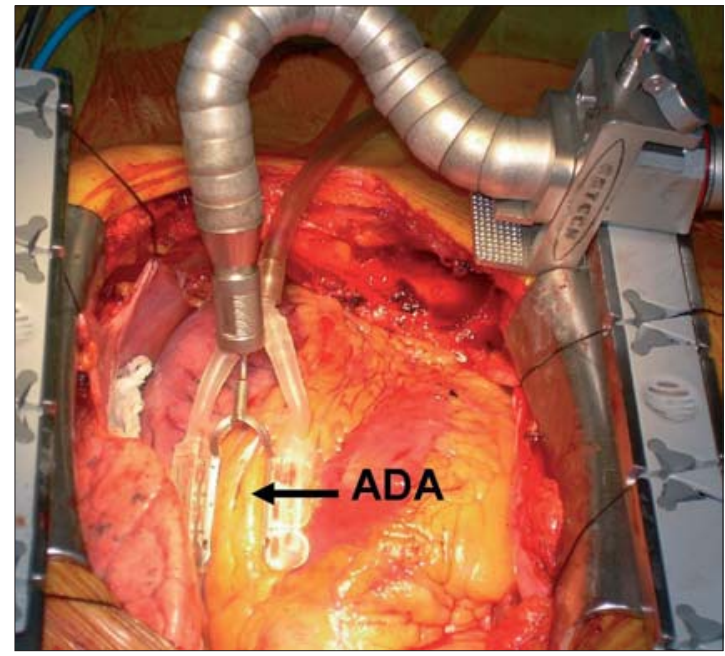

Figura 1. Estabilización de arteria descendente anterior. ADA: arteria descendente anterior.

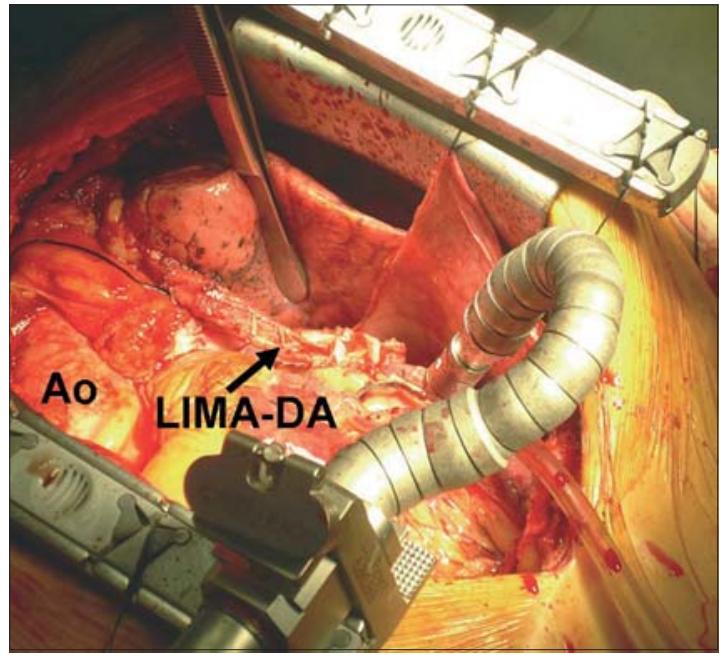

Figura 2. Anastomosis de la arteria mamaria interna izquierda a la arteria descendente anterior finalizada. Ao: aorta ascendente; LIMA-DA: arteria mamaria interna izquierda anastomosada a la arteria descendente anterior. 


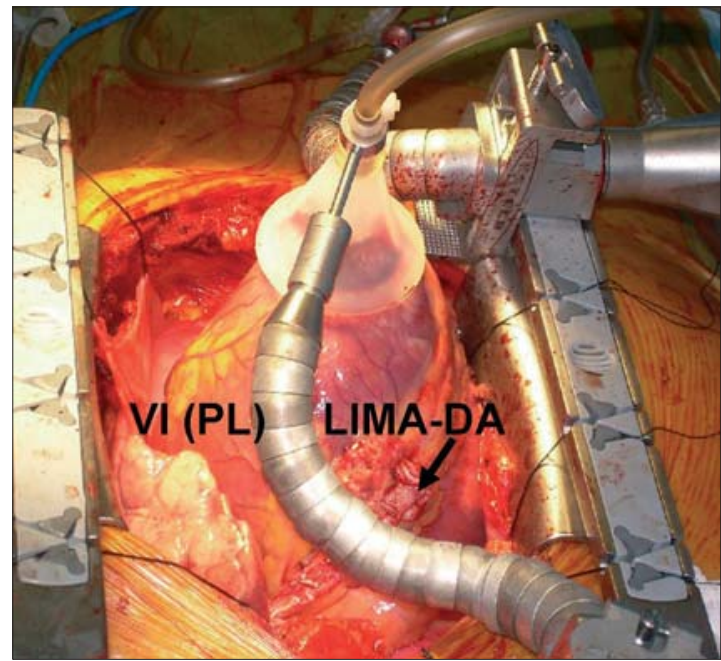

Figura 3. Exposición de la pared lateral. VI (PL): ventrículo izquierdo-pared lateral; LIMA-DA: arteria mamaria interna izquierda anastomosada a la arteria descendente anterior.

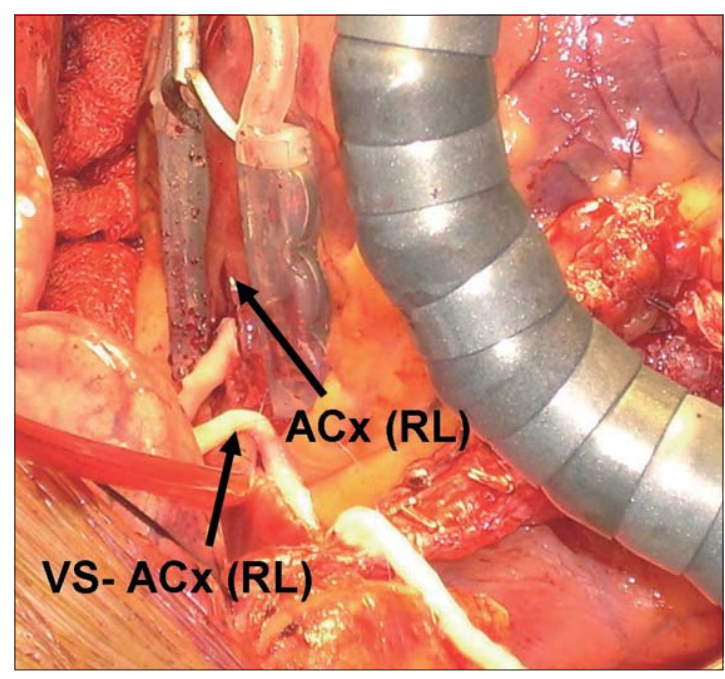

Figura 5. Puente venoso anastomosado a una rama lateral de la arteria circunfleja. ACx (RL): arteria circunflejarama lateral; VS-ACx (RL): vena safena anastomosada a una rama lateral de la arteria circunfleja.

esternón se afronta con puntos de acero inoxidable y los tejidos blandos se sutura con material reabsorbible.

\section{Comentario}

La cirugía sin CEC es una técnica demandante, requiere de una curva de aprendizaje y su aplica-

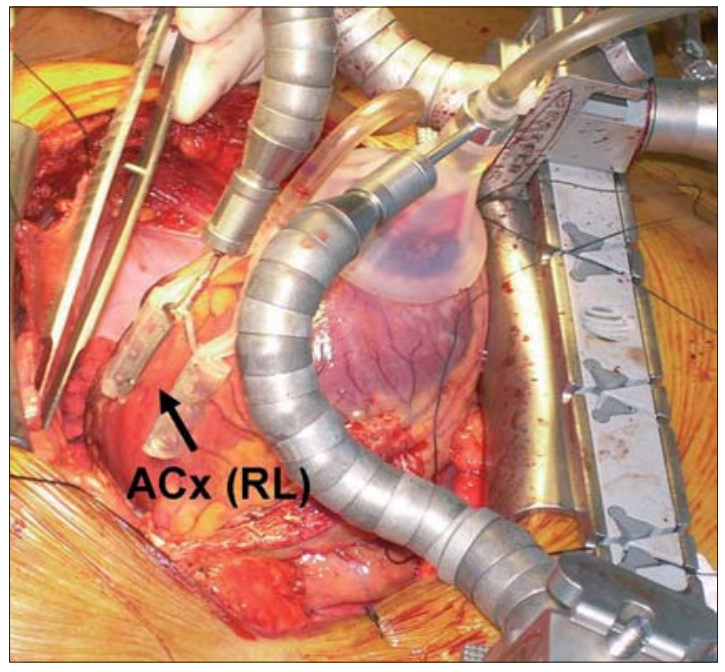

Figura 4. Estabilización de una rama lateral de la arteria circunfleja. ACx (RL): arteria circunfleja-rama lateral.

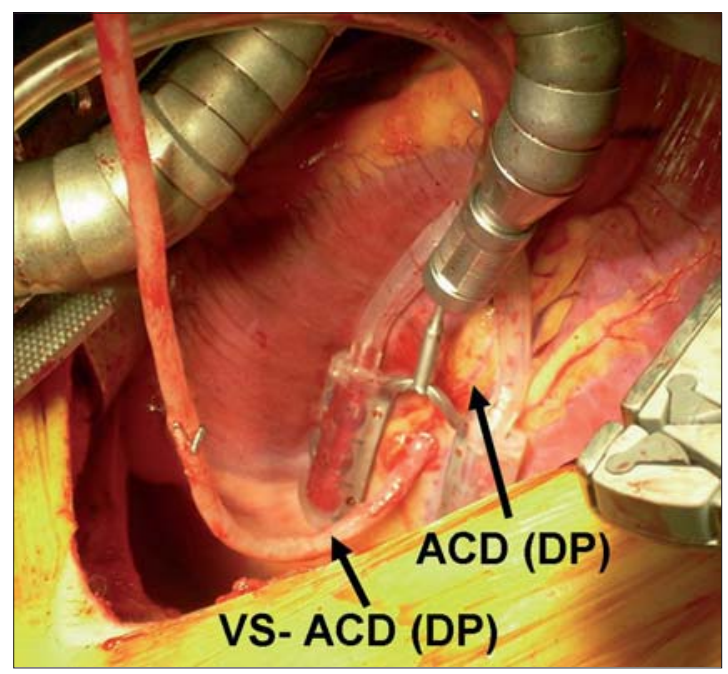

Figura 6. Puente venoso anastomosado a la arteria descendente posterior rama de la arteria coronaria derecha. ACD (DP): arteria coronaria derecha-rama descendente posterior; VS-ACD (DP): vena safena anastomosada a la arteria descendente posterior rama de la arteria coronaria derecha.

ción en todos los pacientes tiene limitaciones, por ejemplo, en corazones hipertróficos, reoperaciones con pericarditis, arterias intramiocárdicas y la presencia de isquemia o inestabilidad hemodinámica ${ }^{8}$. Distintos equipos quirúrgicos señalan que entre el $20 \%$ y $30 \%$ de las cirugías coronarias se pueden realizar con esta técnica ${ }^{8-10}$.

Estudios aleatorios y observacionales (del "mun- 
do real"), señalan que la cirugía sin CEC se asociaría a una reducción en la estadía hospitalaria y a menores costos que la cirugía con CEC. Si bien no se ha demostrado un beneficio en la mortalidad en pacientes de bajo riesgo, existirían beneficios en algunos grupos de pacientes, como aquellos con insuficiencia renal, diabéticos y pacientes con alto riesgo de accidentes neurológicos ${ }^{11-15}$. Los resultados alejados son comparables entre ambas técnicas, excepto por la necesidad de revascularización, que favorecía a la cirugía con $\mathrm{CE}{ }^{9,16}$.

Las ventajas de esta técnica sobre la cirugía con CEC aún son motivo de estudio y discusión. Estudios multicéntricos, prospectivos y randomizados como el CORONARY, que actualmente está en curso, permitirán posiblemente dar respuestas a las múltiples interrogantes que aún existen.

En nuestro equipo hemos ido incrementando el número de cirugías sin $\mathrm{CEC}$, a la fecha hemos realizado alrededor de 250 casos y actualmente la experiencia obtenida nos ha permitido su aplicación a pacientes de mayor riesgo, con más vasos comprometidos y candidatos a un mayor número de puentes. Los resultados publicados de nuestros 100 primeros casos nos permiten señalar que la cirugía coronaria sin CEC en nuestro medio, es una técnica segura y reproducible de revascularización miocárdica $^{17}$

\section{Referencias}

1. Yusuf S, Zucker D, Peduzzi R, Fisher LD, Takaro T, Kennedy JW, et al. Effect of coronary artery bypass surgery on survival: overwiew of 10-years results from randomised trials by the coronary artery bypass graft surgery trialists collaboration. Lancet 1994; 344: 563570 .

2. Kolessov VI. Mammary artery coronary anastomosis as method of treatment of angina pectoris. J Thorac Cardiovasc Surg 1967; 54: 535-544.

3. Trapp WG, Bisarya R. Placement of coronary artery bypass graft without pump oxigenator. Ann Thorac Surg 1975; 19: 1-9.

4. Ankeny JL. To use or not to use the pump oxigenator in coronary bypass operations. Ann Thorac Surg 1975; 19: 108-109.

5. Buffolo E, Andrade JC, Succi J, Leão LE, Gallucci C. Direct myocardial revascularization without cardiopulmonary bypass. Thorac Cardiovasc Surg 1985; 33: 26-29.
6. Buffolo E, Andrade JC, Branco JN, Aguiar LF, Ribeiro $\mathrm{EE}$, Jatene AD. Myocardial revascularization without extracorporeal circulation. Seven-years experience in 593 cases. Eur J Cardiovasc Surg 1990; 4: 504-507.

7. Benetti FJ, Naselli G, Word M, Geffner L. Direct myocardial revascularization without extracorporeal circulation. Experience in 700 patients. Chest 1991; 100: 312-316.

8. Buffolo E, Branco JN, Gerola LR, Aguiar LF, Teles CA, Palma JH et al. Off-pump myocardial revascularization: Critical analysis of 23 years' experience in 3866 patients. Ann Thorac Surg 2006; 81: 85-89.

9. Hannan EL, Wu C, Smith CR, Higgins RS, Carlson RE, Culliford AT, et al. Off-pump versus on-pump coronary artery bypass graft surgery: differences in short-term outcomes and in long-term mortality and need for subsequent revascularization. Circulation 2007; 116: 1145-1152.

10. Lytle BW, Sabik JF. On-pump and off-pump bypass surgery: tools for revascularization. Circulation 2004; 109: 810-812.

11. Nathoe HM, van Dijk D, Jansen EW, Suyker WJ, Diephuis JC, van Boven WJ, et al. A comparison of on-pump and off-pump coronary bypass surgery in low-risk patients. N Engl J Med 2003; 348: 394-402.

12. Stallwod MI, Grayson AD, Mills K, Scawn ND. Acute renal failure in coronary artery bypass surgery: independent effect of cardiopulmonary bypass. Ann Thorac Surg 2004; 77: 968-972.

13. Beauford RB, Goldstein DJ, Sardari FF, Karanam R, Luk B, Prendergast TW, et al. Multivessel off-pump revascularization in octogenarians: early and midterm outcomes. Ann Thorac Surg 2003; 76: 12-17.

14. Srinivasan AK, Grayson AD, Fabri BM. On-pump versus off-pump coronary artery bypass grafting in diabetic patients: a propensity score analysis. Ann Thorac Surg 2004; 78: 1604-1609.

15. Cheng DC, Bainbridge D, Martin JE, Novick RJ. Does off-pump coronary artery bypass reduce mortality, morbidity, and resource utilization when compared with conventional coronary artery bypass? A meta-analysis of randomized trials. Anesthesiology 2005; 102: 188203.

16. Abu-Omar Y, Taggart DP. The present status of offpump coronary artery bypass grafting. Eur J Cardiothorac Surg 2009; 36: 312-321.

17. Seguel E, González R, Stockins A, Alracón E, Neira L. Cirugía coronaria sin circulación extracorpórea: Primeros 100 casos en el Hospital Regional de Concepción. Rev Chil Cardiol 2008; 27: 125-135. 